\title{
EL VALOR TEMPORAL DEL SINTAGMA PREPOSICIONAL LATINO REGIDO POR CUM: APROXIMACIONES DESDE LA TEORÍA DEL LEXICÓN GENERATIVO
}

\author{
MARINA ANABELLA GALLARDO BERG \\ Universidad Nacional del Litoral \\ anabella.gallardoberg@gmail.com
}

Recibido: 15/03/20

Aceptado: $15 / 10 / 20$

Este trabajo busca contribuir a la descripción del sintagma preposicional latino introducido por cum en contextos en que presenta un valor temporal. Exploramos la posibilidad de aplicar el modelo de la Teoría del Lexicón Generativo de PUSTEJOVSKY (1995) para explicar los rasgos subléxicos y los mecanismos generativos que habilitan este valor en el SP. Nuestra propuesta de análisis sostiene que el valor temporal es producto de la acción conjunta, generada por el mecanismo de cocomposición, de los rasgos [-DINAMISMO], [-ORIENTACIÓN] y [+COEXISTENCIA] de la estructura de qualia que presentamos para la preposición cum y los rasgos subléxicos de los nombres eventivos que la preposición toma como complemento.

Latín / Sintagma preposicional / Preposición cum / Valor temporal / Teoría del Lexicón Generativo

THE TEMPORAL VALUE OF THE LATIN PREPOSITIONAL PHRASE INTRODUCED BY CUM: AN APPROACH FROM THE GENERATIVE LEXICON THEORY

This work seeks to contribute to the description of the Latin prepositional phrase introduced by cum in contexts in which it presents a temporal value. We explore the possibility of applying the model of PUSTEJOVSKY's Generative Lexicon Theory (1995) to explain the sublexical features and the generative mechanisms that enable this value in the prepositional phrase. Our analysis argues that the time value is product of the joint 
action, generated by the co-composition mechanism, of the features [-DYNAMISM], [ORIENTATION] and [+COEXISTENCE] of the qualia structure that we present for the preposition cum and the sublexical features of the eventive nouns that the preposition takes as complement.

Latin / Prepositional phrase / Preposition cum / Temporal value / Generative Lexicon Theory

\section{Introducción}

Las preposiciones en latín, como en gran cantidad de lenguas, constituyen un dominio entre el léxico y la sintaxis. De allí que puedan presentar una semántica tan amplia en diferentes contextos discursivos. En la entrada para la preposición cum, el Oxford Latin Dictionary (1968, p. 467) lista quince acepciones semánticas. Estas se pueden resumir en las nociones de compañía (1.a) y (1.b); modo, "expression of manner", en casos como el de (1.c); circunstancia (1.d) y noción de tiempo simultáneo, "simultaneously with, at (a point of time), at the same time or contemporaneusly with" en datos como (1.e).

$(1)^{1}$ a. cum Pansa vixi in Pompeiano. (Cic. Att. 14. 20. 4)

Con Pansa viví en Pompeya.

b. cum omnibus copiis ad Caesarem prervenit. (Caes. Gal. 7. 62. 10)

Se dirige con todas sus tropas a reunirse con César.

c. cum bona venia, quaeso, audiatis, patres conscripti, id quod inuitus eloquar. (Liv. 29. 17. 6)

Por favor, senadores, escuchen con favorable benevolencia esto que digo contra mi voluntad.

d. ibi inexplorato profectus in insidias praecipitat multaque cum caede suorum aegre in apertos campos emersit. (Liv. 21. 25. 9)

Avanzando sin un reconocimiento, cae en una emboscada y, con la masacre de los suyos, angustiosamente logró salir a los campos abiertos.

e. ad portum hinc abii mane cum luci simul; (Pl. Mer. 255)

Por la mañana, con la primera luz del alba, me fui de aquí al puerto.

Las gramáticas tradicionales coinciden en asignar a la preposición cum un valor principal de concomitancia. Junto a este, aparecen los valores de modo, circunstancia y, descripto de manera más o menos detallada, el valor temporal. El presente trabajo busca contribuir a la descripción del valor temporal del sintagma preposicional encabezado por cum, explorando la posibilidad de aplicar el modelo de la Teoría del 
Lexicón Generativo para explicar la combinatoria de rasgos subléxicos que habilita este valor en el SP. Los datos de nuestro corpus han sido tomados de diferentes autores del periodo clásico, y algunos del periodo preclásico: obras de César, Cicerón, Livio, Plauto, Terencio, Mela y Salustio.

El trabajo se organiza del siguiente modo. En el apartado 2 revisaremos brevemente algunas perspectivas de análisis del sintagma preposicional regido por cum cuando presenta un valor temporal ${ }^{2}$. En el apartado 3 nos referiremos a la Teoría del Lexicón Generativo de PusTEJOvSKY (1995), marco teórico desde el cual se enfoca nuestra propuesta. Finalmente, en el apartado 4 presentaremos un análisis para los datos en los cuales el SP encabezado por cum presenta un valor temporal, y en el 5 expondremos nuestras conclusiones.

\section{Perspectivas de análisis del valor temporal del sintagma preposicional regido por cum en la literatura especializada}

SANTESSON (1921) estudia la preposición latina cum y sus equivalentes en otras lenguas romances. De acuerdo con este autor, la semántica principal de la preposición es la cercanía en el espacio, la concomitancia, la unión, la compañía (SANTESSON, 1921, p. 9). A partir de este valor central, SANTESSON (1921, p. 15) considera el valor comitativo de cum en relación con el tiempo ("cum comtitatif par rapport au temps"). Así, en construcciones como cum hoc Catone grandiores natu fuerunt (Cic. Brut. 77), la preposición expresa una noción de "contemporaneidad" entre dos seres vivos, y en construcciones como perdidisti me et fidem mecum tuam! (Pl. Merc. 625), cum establece una relación en el tiempo entre "las cosas y los seres vivos" ("des choses par rapport à des êtres vivants", SANTESSON, 1921, p. 16).

Por lo que respecta a las estructuras que son de nuestro interés en este trabajo, aquellas en las que el sintagma preposicional encabezado por cum funciona como adjunto temporal, Santesson se limita a indicar una semántica de relación "entre las cosas" ("des choses entre elles", SANTESSON, 1921, p. 16) y señala que la preposición "reemplaza" al ablativo temporal: "Notez surtout les expressions: cum luce, die, sole, vere, etc., où cum remplace l'ablatif purement temporel ou l'ablatif absolu (luce oriente, etc.)" (SANTESSON, 1921, p. 16). No se ofrece, sin embargo, una descripción pormenorizada de la semántica de estas expresiones ni mayor explicación respecto de las posibilidades de combinatoria léxica que habilitan este valor temporal en el sintagma preposicional. En el capítulo dedicado a revisar la semántica de preposiciones equivalentes a cum en las lenguas romances, Santesson señala que este valor temporal no es muy frecuente en latín, así como tampoco en las lenguas derivadas que se estudian en este trabajo -italiano, español y portugués- (1921, p. 261).

En su Sintaxe Latine, ERNOUT \& THOMAS (1964, p. 87) dedican un apartado al uso del ablativo en rección de la preposición cum. Los autores señalan tres casos que son 
representados por el ablativo latino: el ablativo propiamente dicho, el instrumental y el locativo. Luego, explican, las preposiciones precisan las nociones que corresponden a cada uno de ellos y que la forma casual no permitiría distinguir:

Trois cas concrets sont représentés, comme on l'a vu, dans l'ablatif latin : l'ablatif proprement dit, l'instrumental et le locatif. Aussi un rôle important a-t-il échu aux prépositions pour préciser les notions qui revenaient à chacun d'eux et que la forme casuelle ne permettait plus de distinguer. $A b$, de, ex se lièrent ainsi à l'ablatif proprement dit; cum à l'ablalif-instrumental marquant l'accompagnement; in, sub, etc., à l'ablatif locatif. (ERNOUT \& THOMAS, 1964, p. 79)

De este modo, la preposición cum se vincularía al ablativo instrumental, marcando la noción de compañía (accompagnament). Los diferentes matices que se presentan en (2) se desplegarían de esta noción semántica. En (2.a), "vino con la embajada", cum designaría la persona con la cual se está; en (2.b), "asediar la curia con la espada en la mano", el objeto que se tiene consigo (sin la preposición cum, el ablativo significaría solo "con la espada"); y en (2.c) el SP encabezado cum diluculo tendría una lectura temporal con matiz de "simultaneidad".

(2) a. cum his legatis... venit. (Caes. Gal. 4. 27. 2)

Il vint avec l'ambassade.

b. obsidere cum gladiis curiam. (Cic. Catil. 1. 32)

Assiéger la curie avec l'épée à la main.

c. egone abs te abii... cum diluculo? (Pl. Am. 743)

Ainsi moi, je t'ai quittée au point du jour? (ERNOUT \& THOMAS, 1964:87)

En segundo lugar, ERNOUT $\&$ THOMAS se refieren al ablativo regido por cum en los contextos en que este SP funciona como complemento de verbos: aliquem cum aliquo comparare ('comparar a alguien con alguien'), res est alicui cum aliquo ('tener un asunto con alguien'), etc. El SP cum + ablativo de la circunstancia concomitante, por su parte, es una forma derivada de los empleos anteriores que indica las circunstancias que acompañan a la acción. Se suele encontrar, además, en esta función tanto el ablativo regido por cum como el empleo solo del ablativo: malo (bono) suo o publico "para su mal (su bien)" o "el del Estado" y cum malo (bono) suo. En síntesis, de acuerdo con ERNOUT E THOMAS, el uso de la preposición cum que revisamos en este trabajo aquel en el cual este sintagma tiene una lectura temporal- sería un derivado de cum + ablativo con noción de compañía.

En el capítulo de su autoría dedicado a las preposiciones, en la Sintaxis del latín clásico, BAÑos BAÑos (2009) discute sobre la polisemia de las preposiciones y los 
intentos de sistematización de sus significados tanto por parte de las gramáticas tradicionales como por parte del estructuralismo. En el caso de este último, se ha propuesto que las preposiciones tienen un único sentido central, independientemente del complemento que presente (BRONDAL, 1950, apud BAÑos BAÑos, 2009, p. 312). Por otro lado, desde la perspectiva funcional, en la que se enmarca el trabajo de BAÑos BAÑos, lo que se busca no es tanto recoger el significado básico de la preposición, a modo de poder explicar sus potenciales realizaciones, sino más bien determinar qué significados de un SP han sido gramaticalizados como funciones semánticas diferenciales. De este modo, BAÑos BAÑOS propone un cuadro, adaptado de HERNÁNDEZ CABRERA (1998) ${ }^{3}$, en el que se presentan las funciones semánticas más frecuentes expresadas por los sintagmas preposicionales en latín clásico. Allí se señalan para la preposición cum las funciones semánticas de tiempo, compañía, modo, circunstancia concomitante y, en un empleo raro o poco frecuente (simbolizado en el cuadro por $(+)$ ), la función instrumental. No se profundiza más sobre las construcciones en las que puede encontrarse cada valor del sintagma preposicional encabezado por cum, ni las motivaciones léxicas o sintácticas que posibilitan el valor temporal en particular.

ASENSIO (1998) realiza un estudio del sintagma preposicional regido por cum con el objetivo de determinar si desempeña realmente las funciones comitativa, modal y circunstancial -las cuales se suelen adjudicar a dicha preposición en los manuales de sintaxis tradicionales-, o si solo son subdivisiones no funcionales de una misma función de carácter general. El análisis se basa en datos de Plauto, Tito Livio y Tácito. Sin embargo, la autora deja fuera de su estudio los ejemplos relativos al valor temporal de la preposición cum, como egone aps te abii hinc hodie cum diluculo? (Pl. Am. 743) o ad portum hinc abii mane cum luci simul (Pl. Mer. 255).

La metodología empleada por ASENSIO para su análisis se basa en la gramática funcional de DIK (DIK, 1978, apud ASENSIO 1998). La autora procede a determinar las funciones semánticas de cum a partir del contenido léxico de los lexemas implicados y comprobar luego si los datos de coordinación y yuxtaposición corroboran sintácticamente la clasificación, dado que, en los datos, cum suele estar coordinado con SSNN en ablativo además de algunos adverbios, por lo que la marca formal (preposición + caso) resulta inicialmente un problema.

A partir de los datos que analiza, la autora concluye que el SP regido por cum, cuando funciona como satélite, puede desempeñar tres funciones distintas: compañía, modo y circunstancia. Lo que permite la identificación de la función de compañía frente a las funciones de modo y circunstancia es el rasgo [+CONCRETO] del nombre que contiene el SP, como omnibus Aetolis en Alexamenus cum omnibus Aetolis citato gradu ad regiam occupandam pergit (Liv. 35. 36. 1) (ASENSIO, 1998, p. 43). Por su parte, el rasgo que diferencia la función de modo de la función de circunstancia sería la [+CORREFERENCIALIDAD] que presenta la primera entre el primer argumento del estado 
de cosas al que remiten y el primer argumento de la predicación en la que aparecen. Así, en cum Scaptium non silentio modo, sed cum adsensu etiam audiri animaduertissent (Liv. 3. 72. 1), el referente de los primeros argumentos de audiri y los estados de cosas que expresan silentio y adsensu es el mismo: las personas a las que se dirige Scaptius. En cambio, en Hanno unus aduersus senatum causam foederis magno silentio propter auctoritatem suam, non cum adsensu audientium (Liv. 21. 10. 2), silentio y adsensu remiten a un estado de cosas cuyo primer argumento tiene un referente distinto al del primer argumento de la predicación (ASENSIO, 1998, p. 43). De este modo, a partir de los rasgos [+/-CONCRETO] de los nombres involucrados en el SP y el rasgo de [+CORREFERENCIALIDAD] entre el nombre regido por cum y el nombre que funciona como argumento externo de la preposición, ASENSIO caracteriza las funciones de compañía, modo y circunstancia. No se ofrecen más detalles acerca de los rasgos que aportaría cum a la construcción ni de cómo podría explicarse el valor temporal en los datos de Plauto señalados más arriba, ya que estos quedan fuera del análisis.

En síntesis, los trabajos que hemos revisado en este apartado sobre la preposición cum sostienen que la semántica básica de la preposición es la de concomitancia. Se señala, aunque sin incurrir en una descripción detallada de estas estructuras, que el valor temporal que puede presentar el SP encabezado por esta preposición parece estar relacionado con el valor principal de concomitancia. Queda entonces por describir y explicar cuáles son los rasgos que habilitan el valor temporal en la construcción. Más específicamente, cuál es la combinatoria selectiva de los rasgos de la preposición y los rasgos del complemento que dan lugar a esta semántica temporal. En los apartados siguientes exploraremos la posibilidad de aplicar el modelo de la Teoría del Lexicón Generativo para responder a estos interrogantes.

\section{La Teoría del Lexicón Generativo}

El marco teórico desde el cual dirigiremos nuestra propuesta de análisis para el SP encabezado por cum con valor temporal es la Teoría del Lexicón Generativo (TLG) de PUSTEJOVSKY (1995). Esta teoría, de corte lexicista, afronta tanto el problema de la polisemia y de la aportación del contexto sintáctico a la desambiguación de las expresiones lingüísticas, como también la cuestión de las fronteras borrosas entre conocimiento lingüístico y enciclopédico. Así, tal como señala DE MIGUEL (2009, p. 28), la principal preocupación de la TLG es postular un modelo del lexicón mental "dinámico, flexible y sensible al contexto", que pueda explicar la polisemia de las palabras sin incurrir en múltiples entradas para cada sentido posible -a modo de un lexicón enumerativo- y, en última instancia, responder a la pregunta básica sobre el leguaje en la teoría generativa de cómo el hablante es capaz de generar sentidos, en principio, no finitos, a partir de recursos que sí lo son. 
El centro de la propuesta de PuSTEJOVSKY consiste en el presupuesto de la infraespecificación (underspecification), esto es, que la falta de especificación de las entradas léxicas las capacita para intervenir en diferentes estructuras sintácticas y, por lo tanto, en distintas operaciones de composición semántica. Dicho de otro modo, se propone que los ítems poseen un significado mínimo y, a partir de principios y mecanismos regulares, pueden desencadenar múltiples significados en contexto.

Los ítems, entonces, se encuentran infraespecificados en el léxico y se especifican en contexto, al combinarse con otros cuyos rasgos concuerden y permitan materializar alguna de sus potencialidades semánticas. Si, por el contrario, los rasgos de los ítems que se combinan no concuerdan se puede dar a) un colapso interpretativo, o b) la intervención de un mecanismo de rescate para que los rasgos se vuelvan compatibles y la construcción pueda ser interpretada.

De este modo, la TLG propone, por un lado, un modelo de la estructuración de la información contenida en las entradas léxicas, a saber, cuatro niveles de representación que mencionaremos a continuación, y, por otro, mecanismos generativos de concordancia de rasgos léxicos que conectan dichos niveles de información y permiten explicar la multiplicidad de sentidos de las palabras en el contexto sintáctico.

\subsection{Niveles de representación léxica}

La TLG propone cuatro niveles de representación en los que se estructura la información contenida en la entrada léxica: la Estructura Argumental, la Estructura Eventiva, la Estructura de Qualia y la Estructura de Tipificación Léxica.

En la Estructura Argumental se codifica el número de argumentos de un predicado, la clase semántica a la que pertenecen (ej. [INDIVIDUO], [OBJETO], [EVENTO]) y la manera en que se realizan sintácticamente. Se establece también una distinción entre constituyentes que pueden formar parte del predicado: a) argumentos auténticos (true arguments) que se realizan sintácticamente como en "Argentina perdió el partido" para el verbo perder; b) argumentos por defecto (default arguments) que forman parte del contenido lógico del predicado aunque no siempre se realicen sintácticamente como en "Argentina perdió el partido contra Francia"; c) argumentos en sombra (shadow arguments), aquellos que están semánticamente incorporados a las palabras pero que solo se materializan mediante operaciones de subtipificación, como "Juan enmantecó el pan con margarina"; d) adjuntos auténticos (true adjuncts), elementos opcionales que expresan el tiempo y el espacio en el cual se enmarca un evento pero no forman parte de su representación semántica como en "Argentina perdió el partido contra Francia en Rusia".

La Estructura Eventiva es una novedad de la propuesta de PusTEJOVSKY. La información aspectual constituye un nivel más de la descripción de la palabra, estructurado y jerarquizado. Sus unidades se descomponen en distintas subestructuras 
o subeventos relacionados entre sí por vínculos de ordenación temporal y prominencia relativa. Podemos tener, de este modo, a) Estado (E): un evento simple que se evalúa sin ponerlo en relación con otros, como amar, saber; b) Proceso (P): sucesión de eventos $\left[\mathrm{e}_{1} \ldots \mathrm{e}_{\mathrm{n}}\right]$ identificados en una misma expresión semántica como correr, nadar; c) Transición (T): evento que identifica una expresión semántica [e], evaluada en relación con su oposición [ $\neg$ e] como construir, escribir; la Transición puede estar compuesta por un proceso $\mathrm{P}$ que da lugar a un nuevo estado. Con esta jerarquización es posible dar cuenta de las nociones de logro y realización como parte de la Transición: si en un evento de transición solo se realiza el subevento final, tenemos un logro, como en "el agua hirvió"; si, por el contrario, se realiza el evento complejo (fase de proceso + logro), tenemos una realización, como en "Juan hirvió el agua".

La Estructura de Qualia es la información potencial sobre las características fundamentales de la entidad a que se refiere (objeto o evento). Está estructurada en cuatro roles o qualia que codifican relaciones lógicas. a) Quale constitutivo: codifica la relación entre un objeto y sus partes constituyentes. b) Quale formal: codifica aquello que distingue el objeto dentro de un dominio más extenso. c) Quale télico: codifica el propósito y función del objeto. d) Quale agentivo: codifica factores implicados en el origen o producción de un objeto.

Finalmente, se establece la Estructura de la Tipificación Léxica, cuyo objetivo es describir cómo se relaciona una palabra con otra en el lexicón mental basado en las informaciones contenidas en las estructuras de Qualia.

\subsection{Mecanismos generativos}

PUSTEJOVSKY propone cuatro mecanismos de concordancia de los rasgos del léxico. La Selección, mecanismo canónico que no exige ningún tipo de acomodación especial. La Acomodación es un mecanismo que opera cuando los rasgos del argumento no concuerdan de manera plena con el predicado, pero pueden hacerlo de manera subsidiaria. La Coacción del Tipo denotado por una palabra es un mecanismo que opera cuando un predicado impone un determinado tipo semántico a su argumento. Y finalmente, la Cocomposición, un mecanismo que entra en funcionamiento cuando el tipo de argumento determina el significado de la construcción. El mecanismo de cocomposición implica una actuación conjunta de la información subléxica del argumento y de la palabra rectora (no de una restricción impuesta por esta última como en el caso de la Acomodación y la Coacción de Tipo).

\subsection{Acerca del análisis de preposiciones en el marco de la TLG}

Si bien la TLG se suele aplicar para el análisis de categorías de clases abiertas, como el nombre, el verbo y el adjetivo, se pueden encontrar también trabajos referidos a la polisemia de las preposiciones, como son los de ZATO $(2010,2011)$ y OMORI (2014) 
para preposiciones en español. Estos trabajos consideran, siguiendo a CHOMSKY (1981), que las preposiciones pueden ser unas veces léxicas y otras funcionales y esta distinción es de carácter semántico. ZATO sostiene que el hecho de considerar la preposición como una categoría léxica no debería implicar que tenga gran carga semántica. Este autor considera, más bien, que las preposiciones se asemejan a verbos como hacer, que tiene una intensión baja de significado, y por lo tanto es altamente polisémico como las preposiciones: "Todas las preposiciones se asemejan a este último verbo; son, pues, categorías de naturaleza soporte o ligera, lo que les permite aparecer en multitud de contextos" (ZATO, 2011, p. 45). Adoptar esta perspectiva frente a las preposiciones le permite a ZATO explicar la selección de determinadas preposiciones que hacen los verbos en términos de concordancia de sus rasgos semántico-aspectuales desde la Teoría del Lexicón Generativo de PuSTEJOVSKY. En nuestro trabajo seguiremos la posición de ZATO para explicar el valor de cum.

\section{El valor temporal del sintagma preposicional encabezado por cum: una propuesta de análisis}

Tal como señala ASENSIO (1998) en su trabajo de caracterización funcional del sintagma preposicional encabezado por cum, identificar la función de cum a partir de sus marcas formales (preposición + caso) resulta, inicialmente, problemática ya que la preposición suele estar coordinada con nombres en caso ablativo y, si se trata de identificar su valor temporal, se añade la dificultad de que el latín cuenta también con la conjunción cum que introduce subordinadas temporales. Aquí nos abocaremos a los casos en que el SP encabezado por cum funciona como un adjunto temporal en la construcción, casos que, comparados con las otras funciones de cum señaladas anteriormente, son menos habituales. Los datos de nuestro corpus han sido tomados de diferentes autores del periodo clásico, y algunos del periodo preclásico: obras de César, Cicerón, Livio, Plauto, Terencio, Mela y Salustio. Consideremos, inicialmente, los datos de Plauto:

(4) a. egone aps te abii hinc hodie cum diluculo?" (Pl. Am. 743)

¿Acaso yo me fui de aquí hoy al amanecer?

b. ad portum hinc abii mane cum luci simul; (Pl. Mer. 255)

Me iré de aquí al puerto mañana al amanecer.

Las oraciones de (4.a) y (4.b) presentan un verbo núcleo abii cuya estructura argumental (Figura 1) está especificada por un argumento verdadero (true argument) [+ANIMADO] para la función de sujeto: ego en (4.a) y un sujeto tácito con rasgos de primera persona singular en (4.b). La estructura argumental de abii presenta también argumentos por defecto (default argument) que forman parte del contenido lógico de 
este evento, aunque no necesariamente se realicen: lugar desde (aps te / hinc) y hacia donde se parte (este último realizado solo en (4.b) con ad portum), locus unde y locus quo respectivamente. Se realizan también los adjuntos cum diluculo (4.a) y cum luci simul (4.b) que aportan una especificación de circunstancia temporal al evento.

$$
\left[\begin{array}{l}
\text { abeo } \\
\text { ESTR.ARG }=\left[\begin{array}{l}
\text { ARG1 }=\text { x:animado } \\
\text { ARG-D1= locus_unde } \\
\text { ARG-D2= locus_quo } \\
\text { ARG-D3= medio } \\
\text { ADJ1= tempo } \\
\text { ADJ2= compañia }
\end{array}\right]
\end{array}\right]
$$

Figura 1

Podemos observar que en los dos casos de (4) lo que privilegia la lectura temporal del SP encabezado por cum es el complemento de la preposición. En la oración de (4.a), diluculo ('el amanecer') es un nombre que posee estructura eventiva en su representación semántica (Figura 2), una transición, de la cual, en el SP cum diluculo ('con el amanecer'), se focaliza el subevento $e_{1}$, esto es, el proceso, el momento en que está amaneciendo.

$$
\left[\begin{array}{l}
\text { diluculum } \\
\text { ESTR.ARG }=[\mathrm{ARG} 1=\mathrm{x}: \text { top }] \\
\text { ESTR.EVEN }=\left[\begin{array}{l}
\mathrm{E} 1=\text { e } 1 \text { :proceso } \\
\mathrm{E} 2=\mathrm{e} 2 \text { :estado }
\end{array}\right] \\
\cdots
\end{array}\right]
$$

Figura 2

En el caso de (4.b), el término de la preposición cum, luci, no es un nombre eventivo; sin embargo, en este contexto sintáctico, la interpretación que emerge es análoga a "con la salida del sol" / "con el día”, con lo que parece enfocar, además, un punto ingresivo, "el comienzo del día". De este modo, luci guarda una relación interléxica de metonimia con ítems como die, codificada en su qualia constitutivo como "parte_día". A partir de esta información subléxica puede operar un mecanismo que coacciona el ítem (mecanismo de inserción léxica de coacción de tipo) para volverlo interpretable en el sentido requerido de la construcción, esto es, como un nombre que denota un evento y por esto aporta un valor temporal al SP.

Hechas estas observaciones iniciales, cabe preguntarnos cuál es la naturaleza de cum y si la preposición aporta carga semántica o aparece más bien desemantizada. 
Junto a estas preguntas, debemos señalar que, tanto en (4.a) como en (4.b), el SP aporta un matiz temporal de simultaneidad entre el evento denotado por el nombre término de la preposición y el evento denotado por el verbo de la oración. Se focaliza, además, un subevento puntual del complemento de la preposición, tal como lo hemos observado con diluculo y luci. Antes de responder a las preguntas acerca de la carga semántica que aporta cum a la estructura, consideremos algunos datos más:

(5) a. ceterum ego rus cras cum filio / cum primo luci ibo hinc. (Ter. Ad. 840-41) Por lo demás, mañana con la primera luz yo me iré de aquí al campo con mi hijo. b. cum prima luce densa nebula saltum omnem camposque circa intexit. (Liv. 26. 17. 12)

Con la primera luz una densa niebla cubre alrededor todo el paso y el campo.

c. Metellus cum prima luce furtim in campum itineribus prope deviis currebat; (Cic. Att. 4. 3. 4)

Al amanecer, Metelo corría furtivamente hacia el campo por caminos desviados.

d. accurisse Romam et cum primo luci Pomponii domum venisse dicitur. (Cic. Off. 3. 112)

Se dice que se apresuró a Roma y con la primera luz se presentó en la casa de Pomponio.

e. et cum die iam sol factus attollitur. (Mela 1. 95)

Y ya al amanecer el suelo hecho se realza.

f. legionem, cum qua hiemabat, et quam plurumos potest Numidas equites pariter cum occasu solis expeditos educit. (Sal. Jug. 68. 2)

Con el ocaso del sol, hace salir a la legión, con la cual pasaba el invierno, y asimismo a cuantos más jinetes númidas puede sin equipaje.

g. Perseus quoque, cum aduentu consulis simul et ueris principio strepere omnia mouerique apud hostes uelut nouo bello cerneret. (Liv. 44. 34. 10)

Perseo del mismo modo, notaba que, con la llegada del cónsul y al mismo tiempo el principio de la primavera, todo cerca de los enemigos resonaba con estrépito y se movía como en una nueva guerra.

En las oraciones de (5) podemos observar una recurrencia de los puntos que hemos comentado acerca de las oraciones de (4). El SP encabezado por cum funciona, en cada caso, como adjunto temporal (true adjunct) que aporta un matiz de simultaneidad entre la estructura eventiva del complemento de la preposición y el evento denotado por el verbo principal de la oración, focalizando un punto ingresivo: cum die, cum primo luci, cum occasu solis. Aquello que privilegia la lectura temporal de los SSPP son, como señalábamos anteriormente, los nombres eventivos complementos de la preposición. En (5.e), die es un nombre eventivo simple, con una estructura eventiva 
de proceso; en el SP cum die se focaliza su punto inicial, razón por la que (5.e) puede traducirse como "al amanecer". Los casos de (5. a, b, c y d) son análogos a (4.b), presentando además el modificador primo/prima que define aún más el aspecto ingresivo.

En el caso de (5.f), occasu es un nombre deverbal, que conserva la estructura eventiva del verbo occido, una transición constituida por un $\mathrm{e}_{1}$ : proceso y un $\mathrm{e}_{2}$ : estado. En el SP se focaliza el subevento inicial $e_{1}$, "cuando comienza a caer el sol". En (5.g), los complementos (coordinados) de la preposición son adventu consulis ("la llegada del cónsul") y veris principio ("el principio de la primavera"). El primer caso se trata, al igual que occasu, de un nombre deverbal que conserva la estructura eventiva del verbo advenio. En el sintagma veris principio, la estructura eventiva de proceso es aportada por el complemento genitivo veris y el nombre principio delimita su fase inicial.

En síntesis, el valor temporal del SP encabezado por cum se caracteriza por la presencia de un nombre con estructura eventiva como complemento de la preposición, y la construcción focaliza, además, un punto ingresivo del evento denotado por el nombre. Trataremos ahora de responder a las preguntas formuladas arriba y aportar una propuesta de análisis para el valor temporal de cum.

Desde el enfoque que adoptamos aquí, entendemos a cum como una preposición léxica y, por lo tanto, tiene su respectiva representación semántica. Siguiendo a ZATO (2010, 2011) en su análisis de la preposición española con, proponemos la siguiente estructura de qualia (Figura 3) para la preposición cum.

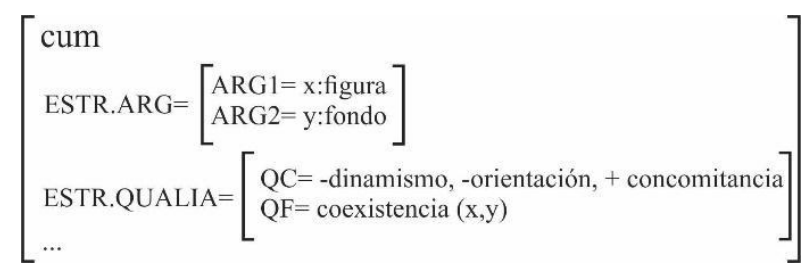

Figura 3

En tanto preposición, cum establece una relación binaria entre dos entidades (incluyendo eventos): una Figura y un Fondo. La Figura, en términos de TALMY (2000, p. 312), es la entidad, objeto o sustancia que está situada o en movimiento, mientras que el Fondo es la ubicación, objeto o sustancia respecto de la cual la trayectoria, orientación o sitio de la Figura es caracterizado. La Figura es siempre un elemento externo al sintagma preposicional, mientras que el Fondo es el complemento de la preposición. En la estructura de Qualia tenemos un quale constitutivo (cuya función es la de codificar la relación entre un objeto y sus partes constituyentes) con rasgos de [-DINAMISMO], [-ORIENTACIÓN] y [+CONCOMITANCIA], así como un quale formal (que codifica aquello que distingue el objeto dentro de un dominio más extenso) de [+COEXISTENCIA]. El rasgo de [+CONCOMITANCIA] refiere a que cum denota una 
coincidencia entre la Figura y el Fondo, en espacio o tiempo. De este rasgo se deriva el valor más habitual de cum: la compañía. En Cum Pansa vixi in Pompeiano (Cic. Att. 14. 20. 3. 12), el sujeto tácito con rasgos de primera persona singular (Figura) coincide con Pansa en un espacio, Pompeiano (Fondo). En ibi inexplorato profectus in insidias praecipitat multaque cum caede suorum aegre in apertos campos emersit (Liv. 21. 25. 9. 2), dado el contexto sintáctico, se habilita, en cambio, la lectura de circunstancia concomitante. De este modo, la preposición cum estaría así infraespecificada y la gama de sentidos que puede adquirir el SP encabezado por cum en la sintaxis estaría dada por las combinaciones posibles con otros ítems léxicos, regidas por los principios y mecanismos generativos que propone la TLG.

La lectura temporal que hemos observado en (4) y (5) para el SP encabezado por cum es el resultado del mecanismo de cocomposición que entra en funcionamiento cuando el tipo de argumento determina el significado del predicado. Tal como lo explicábamos en el apartado 3.2, este mecanismo implica una actuación conjunta de la información subléxica del ítem rector y su argumento en la generación del significado. Así, la concordancia de los rasgos de la preposición cum y los rasgos subléxicos de los nombres eventivos actúan de forma conjunta habilitando la lectura temporal de un momento puntual simultáneo al evento denotado por el predicado. Los rasgos de [-DINAMISMO], [-ORIENTACIÓN] del quale constitutivo cum son responsables por marcar ese "momento puntual": focalizan el subevento e1 de la estructura eventiva del complemento de la preposición. El rasgo [+COEXISTENCIA] del quale formal especifica la simultaneidad entre el evento del complemento de la preposición (Fondo) y el evento denotado por el verbo (Figura) que toma el sintagma preposicional como adjunto.

\section{Conclusiones}

En este trabajo hemos abordado el análisis del valor temporal del SP encabezado por cum, valor con el que se suele presentar a la preposición cum en la literatura general y especializada, aunque sin la atención analítica que se puede encontrar para los otros valores con los que se caracteriza a esta preposición. Nuestro objetivo fue contribuir a la descripción y explicación de la combinatoria selectiva de rasgos de la preposición y los rasgos del complemento que dan lugar a esta semántica temporal del sintagma preposicional.

Hemos propuesto un análisis desde la Teoría del Lexicón Generativo, enfoque que nos permitió presentar una representación semántica infraespecificada para la preposición cum. La semántica de la preposición se especificará o determinará en contexto, al combinarse con otros ítems léxicos con cuyos rasgos concuerde a partir de mecanismos generativos. Para el caso del valor temporal, que nos ocupa en este trabajo, hemos concluido que dicha interpretación se debe al accionar del mecanismo 
de cocomposición, a partir del cual el significado temporal es producto de la acción conjunta de los rasgos subléxicos de los nombres eventivos y los rasgos [-DINAMISMO], [-ORIENTACIÓN] y [+COEXISTENCIA] de la preposición cum. Al combinarse la preposición cum con un nombre eventivo, se focaliza el subevento inicial de este último, obteniendo un aspecto ingresivo.

El modelo de la TLG, tal como lo señala De MiguEL (2009, p. 30), constituye una propuesta muy sugerente para revisar los problemas clásicos de semántica léxica desde una perspectiva innovadora que ha de tener, además, consecuencias en la lexicografía ya que permite postular entradas menos específicas y, en adición, ofrece instrumentos para la explicación de las distintas acepciones que puede tener un término en contexto. Con todo, el análisis que hemos expuesto aquí podrá detallarse y mejorarse en trabajos futuros que tomen en consideración un corpus más amplio de estas estructuras. Del mismo modo, la representación semántica de cum deberá ser evaluada y pulida con análisis en los que se aborde otros valores de la preposición, desde esta perspectiva.

\section{Bibliografía}

ASENSIO, P. (1998). Caracterización funcional de cum + ablativo". En Actas del IX Congreso Español de Estudios Clásicos, vol III (pp. 37-44). Ediciones Clásicas.

Baños BaÑos, J.M. (2009). Capítulo XI. Preposiciones. En Sintaxis del latín clásico. Liceus, Servicios de Gestión y Comunicación S.L.

CHOMSKY, N. (1981). Lectures on Government and Binding. Foris.

De Miguel, E. (2009). La teoría de lexicón generativo. En E. DE Miguel (ed.), Panorama de la lexicología. Ariel.

GLARE, P.G.W. (1968). Oxford Latin Dictionary. Oxford University Press/Clarendon Press.

DIK, S. C. (1978). Functional Grammar. Foris.

ERNOUT, A. \& THOMAS, F. (1964). Sintaxe latine. Éditions Klincksieck.

HERNÁNDEZ CABRERA, T. (1998). Las preposiciones latinas en época clásica. Estudio funcional (Tesis doctoral, Universidad de La Laguna). Inédita.

OMORI, H. (2014). ¿Cómo explicar la polisemia de las preposiciones en el lexicón generativo? Una propuesta para la enseñanza del español desde la teoría lingüística. En Actas de XXIV Congreso Internacional de ASELE (Asociación para la Enseñanza de Español como Lengua Extranjera), 543-550.

PusteJovsky, J. (1995). The Generative Lexicon. MIT press.

SANTESSON, C.G. (1921). La particule cum comme préposition dans les langues romanes. Édouard Champion. 
TALmY, L. (2000). Toward a Cognitive Semantics, Vol I: Concept structuring systems [Language, Speech, and Communication]. The MIT Press.

ZATO, Z. (2010). Las preposiciones del español: una aproximación subléxica (Tesina de fin de máster, Universidad Autónoma de Madrid). En prensa.

ZATO, Z. (2011). Alternancia preposicional en los complementos preposicionales regidos en español: Aproximacion sublexica. Círculo de Lingüística Aplicada a la Comunicación, 48, 41-76.

\section{Notas}

${ }^{1}$ Los ejemplos son tomados del Oxford Latin Dictionary (1968). Las traducciones al español son nuestras.

${ }^{2}$ La revisión no pretende ser exhaustiva, dado que el objetivo es apenas mostrar algunas descripciones que se han dado sobre este valor de la preposición y los rasgos que lo habilitan.

${ }^{3}$ Llama la atención que en el trabajo de HERNÁNDEZ CABRERA no se considera el valor temporal para la preposición cum, solo el de compañía y modo (HERNÁNDEZ CABRERA, 1998, p. 294). 